NASA Technical Memorandum 100287

\title{
Case Study of Active Array Feed Compensation With Sidelobe Control for Reflector Surface Distortion
}

R.J. Acosta, A.J.M. Zaman, E.A Bobinsky Lewis Research Center

Cleveland, Ohio

and

A.R. Cherrette and S.W. Lee

University of Illinois at Urbana-Champaign

Urbana, Illinois

Prepared for the

1988 AP-S/URSI International Symposium

sponsored by the Institute of Electrical and Electronics Engineers

Syracuse, New York, June 6-10, 1988

\section{NMSA}


CASE STUDY OF ACTIVE ARRAY FEED COMPENSATION WITH

\title{
SIDELOBE CONTROL FOR REFLECTOR SURFACE DISTORTION
}

\author{
R. Acosta, A. Zaman, E. Bobinsky \\ National Aeronautics and Space Administration \\ Lewis Research Center \\ Cleveland, Ohio 44135 \\ A.R. Cherrette and S.W. Lee \\ Electromagnetic Laboratory \\ University of Illinois at Urbana-Champaign \\ Urbana, Illinois 61801
}

\section{SUMMARY}

The feasibility of electromagnetically compensating for reflector surface distortions has been investigated. The performance characteristics (gain, sidelobe levels, etc.) of large communication antenna systems degrade as the reflector surface distorts mainly due to thermal effects from a varying solar flux. The techniques descibed in this report can be used to maintain the design performance characteristics independently of thermal effects on the reflector surface. With the advent of monolithic microwave integrated circuits (MMIC), a greater flexibility in array-fed reflector system design can be achieved. MMIC arrays provides independent control of amplitude and phase for each of many radiating elements of the feed array. It is assumed that the surface characteristics ( $x, y, z$, its first and second derivatives) under distortion condition are known.

\section{INTRODUCTION}

The feasibility of electromagnetically compensating for reflector surface distortions has been investigated. The performance characteristics (gain, sidelobe level, null location, beamwidth, etc.) of large communication antenna systems degrade as the relfector surface distorts mainly due to thermal effects from a varying solar flux. The techniques described here can be used to maintain the design performance characteristics independently of thermal effects on the reflector surface. It is assumed that the surface characteristics ( $x, y, z$, its first and second derivatives) under distortion conditions are known. Surface error compensating techniques with an array feed are examined using an offset parabolic reflector geometry and a sinusoidal distortion profile.

\section{SURFACE ERROR COMPENSATION TECHNIQUES}

The goal of surface error compensation can be described as follows: given the desired antenna performance, feed array geometry, and a set of surface points describing the distorted reflector, it is required to determine the feed element excitations (amplitude/phase) that will give rise to the desired antenna performance (gain, sidelobe level, etc.). Basically there are two techniques for obtaining the feed array excitations, namely a transmitting technique (refs. 1 and 2) and a receiving technique (ref. 3). Graphical description of these two approaches are presented in figures 1 and 2 , respectively. 
In the receiving approach the compensation and control of antenna sidelobe level can be realized by providing a correct amount of taper to the incoming plane wave. This approach has the advantage of low sidelobes, however the directivity is not a maximum. But nevertheless it provides a good directive gain (within $2 \mathrm{~dB}$ of ICFM). On the other hand the transmitting approach provides the highest directivity, but also results in high sidelobe levels. Further, optimum directivity can only be achieved by adjusting the individual feed element pattern indices $q E$ and $q H$. A block diagram representation of a receiving algorithm is presented in figure 3. One of the significant features of this implementation is the unique analytical description of the distorted reflector surface. Many numerical techniques (polynomial splines, global and local) for interpolating reflector surface points have been extensively developed in the open literature (ref. 4). These techniques fail to represent the distorted surface in an optimum sense due to the possibility of an infinite number of solutions for describing the specified surface. This alogrithm employs a method in which the discrete set of distorted reflector surface points is best separated into two components, a best fit paraboloid to describe the undistorted surface component and a fourier series expansion of the residual surface error component. The spatial spectrum of the surface error component in a given reflector is unique. Correlation between these spectral components (amplitude and frequency) and the compensating array geometry can be established (ref. 5). It is further shown that the feed array area required to fully compensate for the surface error is a function of the maximum spatial frequency component and the best fit paraboloid geometry.

\section{RESULTS}

The surface error compensation techniques have been demonstrated using the reflector geometry and the distortion profile shown in figure 4 . Figures 5 and 6 show the undistorted and distorted antenna patterns respectively. These cases assume a single feed at the focal point. Note that the antenna boresight directivity has been reduced to $38 \mathrm{~dB}$ in the distorted case. Figures 7 and 8 show the corresponding compensated patterns using the transmitting approach (best directivity) and receiving approach (lowest sidelobe level) respectively. The array configuration used for both cases is depicted in figure 9 . The gain has been recovered within $2 \mathrm{~dB}$ in both cases. The sidelobe levels using the receiving approach were lower by 3 to $5 \mathrm{~dB}$ than the ones produced by the transmitting approach as expected. Computationally the receiving algorithm was very fast (20 min of C.P.U. time - IBM 370) as compared to the transmitting alogorithm (1 hr of C.P.U. time - IBM 370). Above results indicate the computational ease and better sidelobe level control in the DCFM technique and perhaps suggests its application in an adaptive type of implementation involving large reflector antenna systems.

\section{REFERENCES}

1. A.R. Cherrete, P.T.C. Lam, S.W. Lee, and R. Acosta, "Compensation of Distorted Offset Parabolic Reflector Using an Array Feed, "IEEE Interna tional Symposium Digest on Antennas and Propagation, Vol. 2, New York: IEEE, 1986, pp. 515-518. 
2. Y. Rahmat-Sami i, "A Generalized Reflector/Array Surface Compensation Algorithm for Gain and Sidelobe Control," IEEE International Symposium Digest on Antennas and Propagation, Vol. 2, New York; IEEE, 1987, pp. 760-763.

3. R. Acosta, "Compensation of Reflector. Distortions Using Conjugate Field Matching," IEEE International Symposium Digest on Antennas and Propagation, Vol. 1, New York; IEEE, 1986, pp. 259-262.

4. E.C. Ngai, and C.E. Profera, "Application of Bivariate Interpolation to Antenna Related Problems," IEEE Trans. Antennas Propag., Vol. AP-32, pp. 735-739, 1984 .

5. A.R. Cherrete, R. Acosta, P.T. Lam, and S.W. Lee, "Compensation of a Reflector Antenna Surface Distortion Using an Array Feed, "accepted for publication in IEEE Trans. Antennas Propag., 1987.
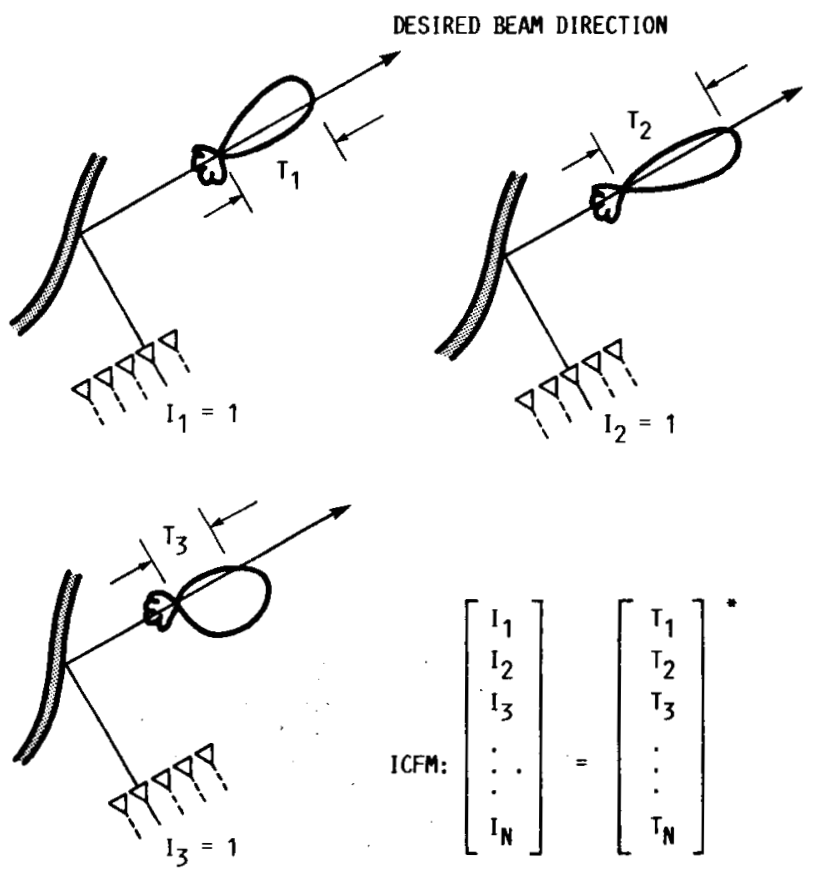

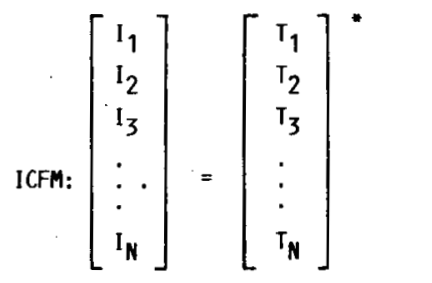

FIGURE 1. - ILLUSTRATION OF INDIRECT CONJUGATE FIELD MATCHING (ICFM) TECHNIQUUE.
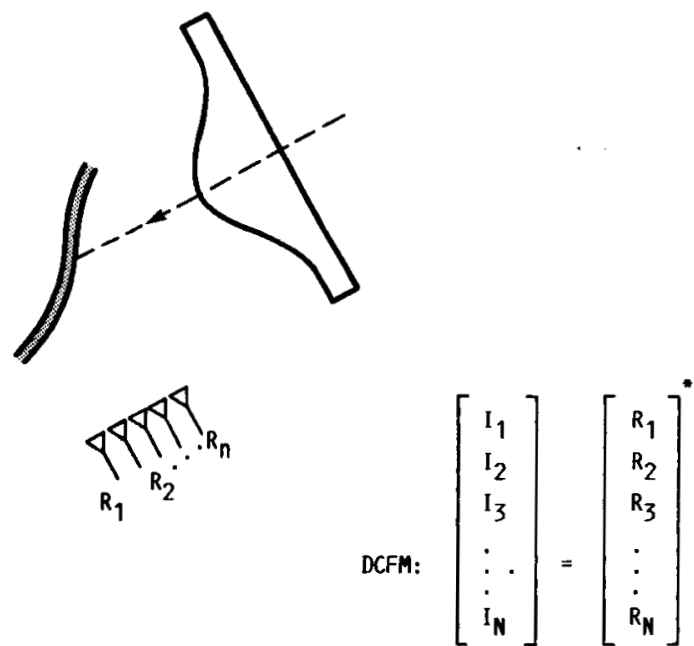

FIGURE 2. - ILLUSTRATION OF DIRECT CONJUGATE FIELD MATCHING (DCFM) TECHMIQUE. 


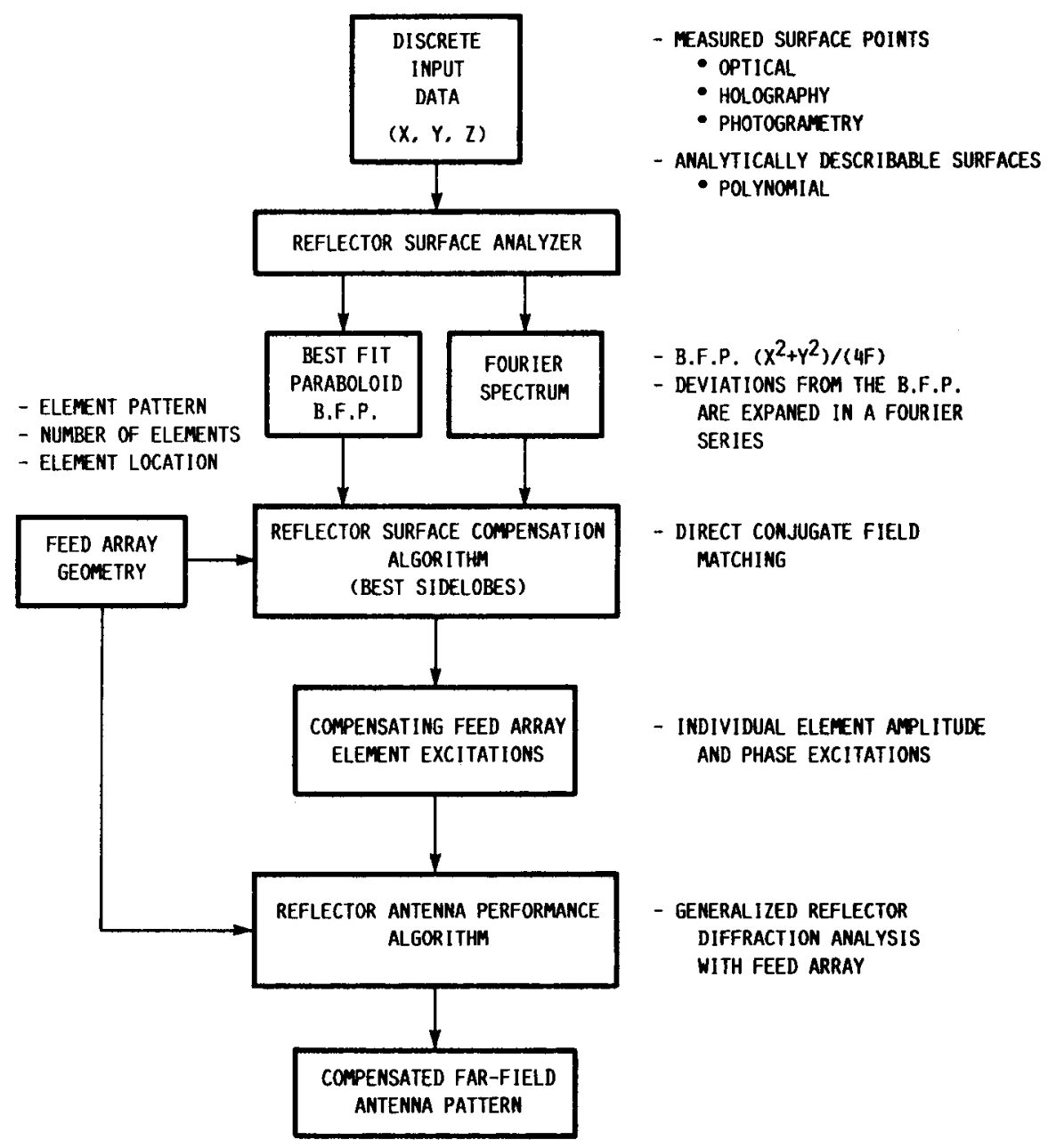

FIGURE 3. - NUMERICAL IMPLEMENTATION OF DCFM.
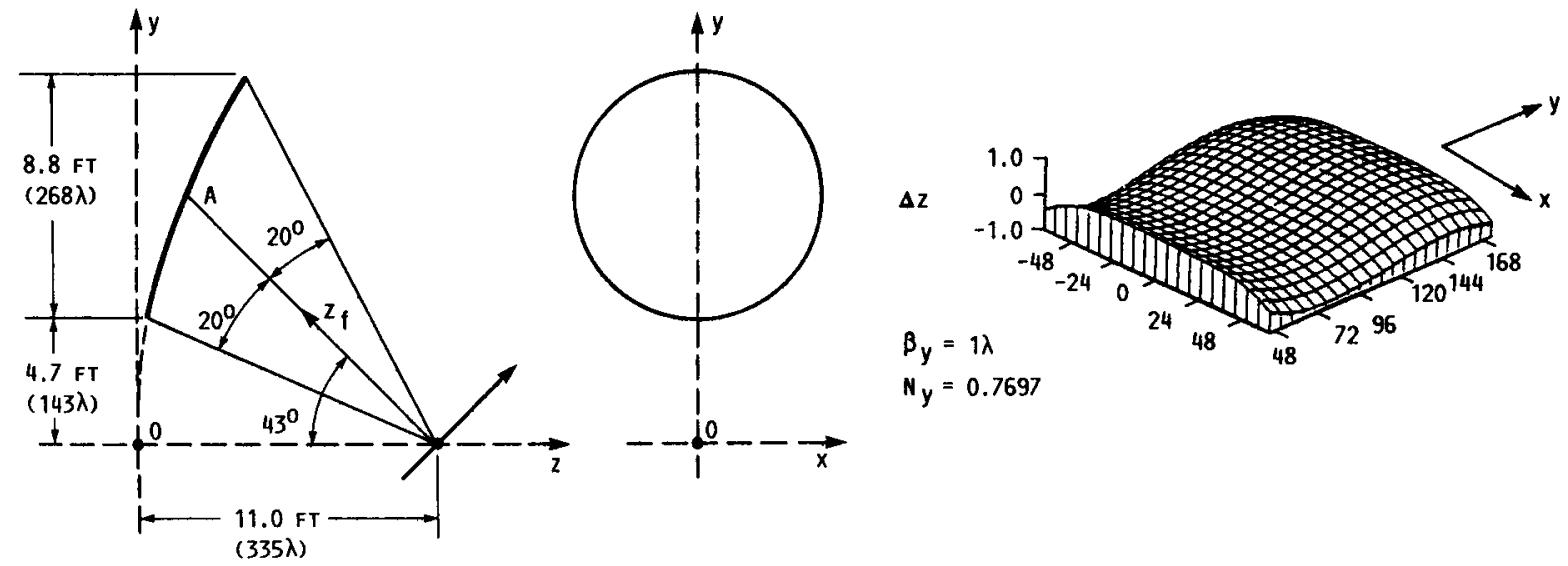

$N_{y}=0.7697$

FIGURE 4. - REFLECTOR ANTENNA GEOMETRY AND DISTORTION PROFILE. 


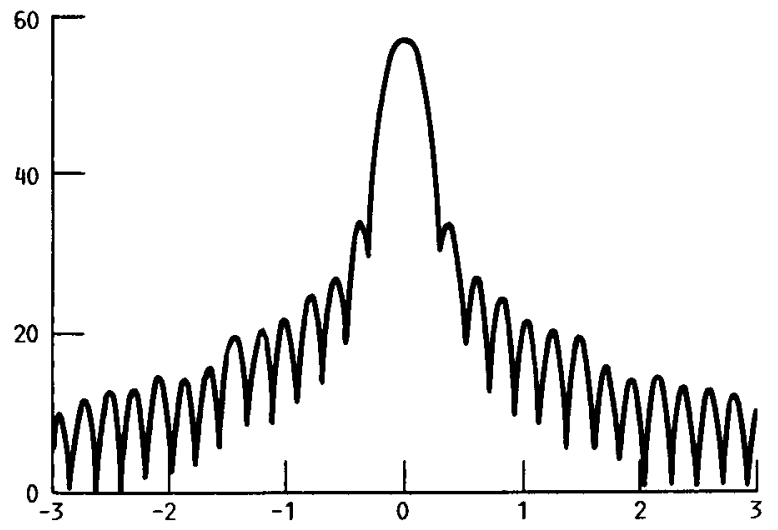

FIGURE 5. - UNDISTORTED ANTENNA PATTERN.

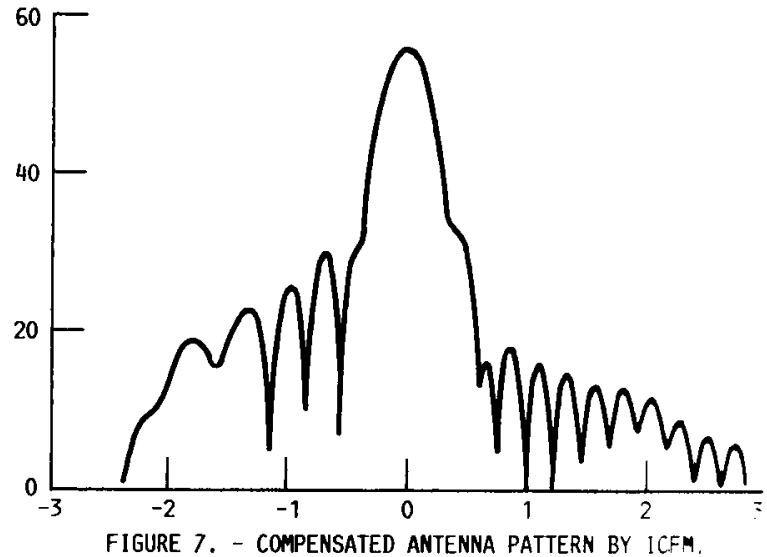

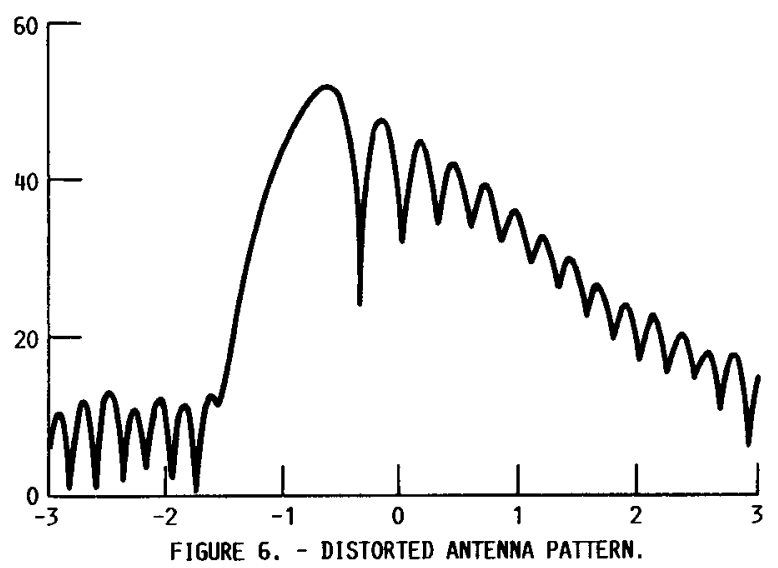

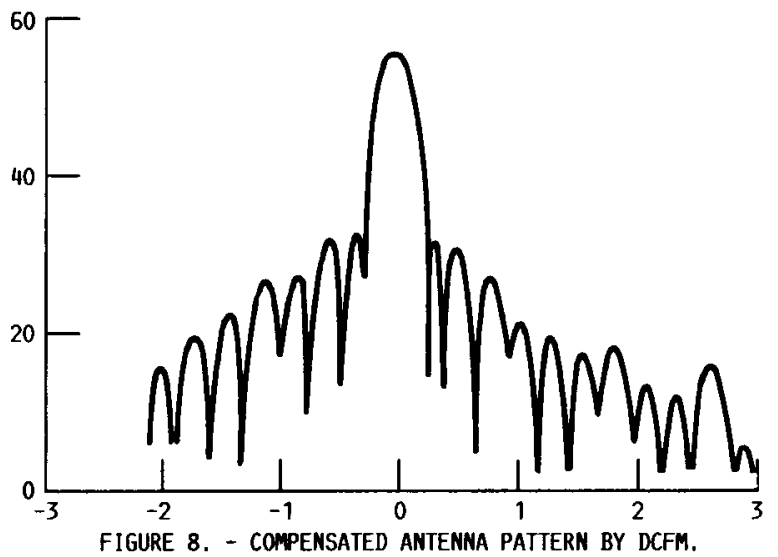

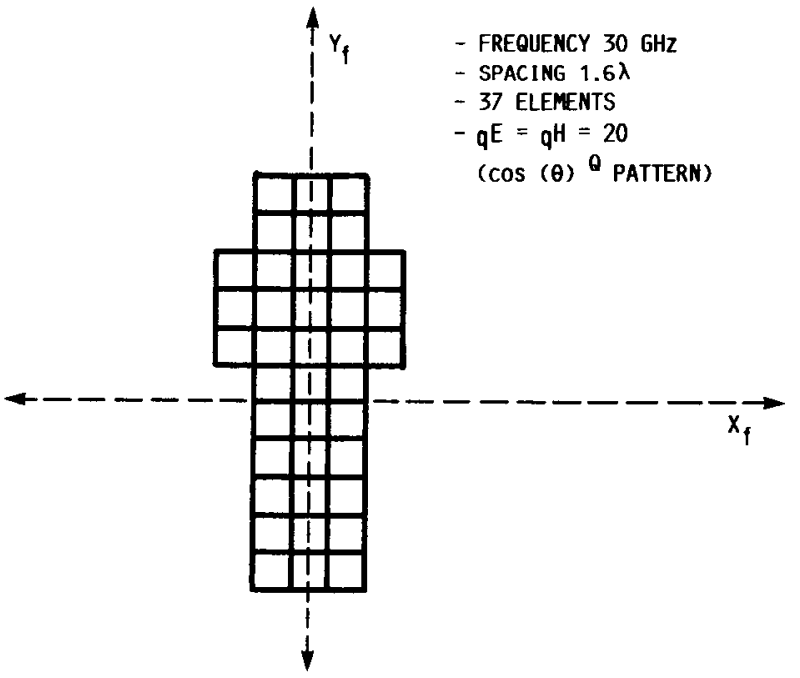

FIGURE 9. - FEED ARRAY GEOMETRY. 


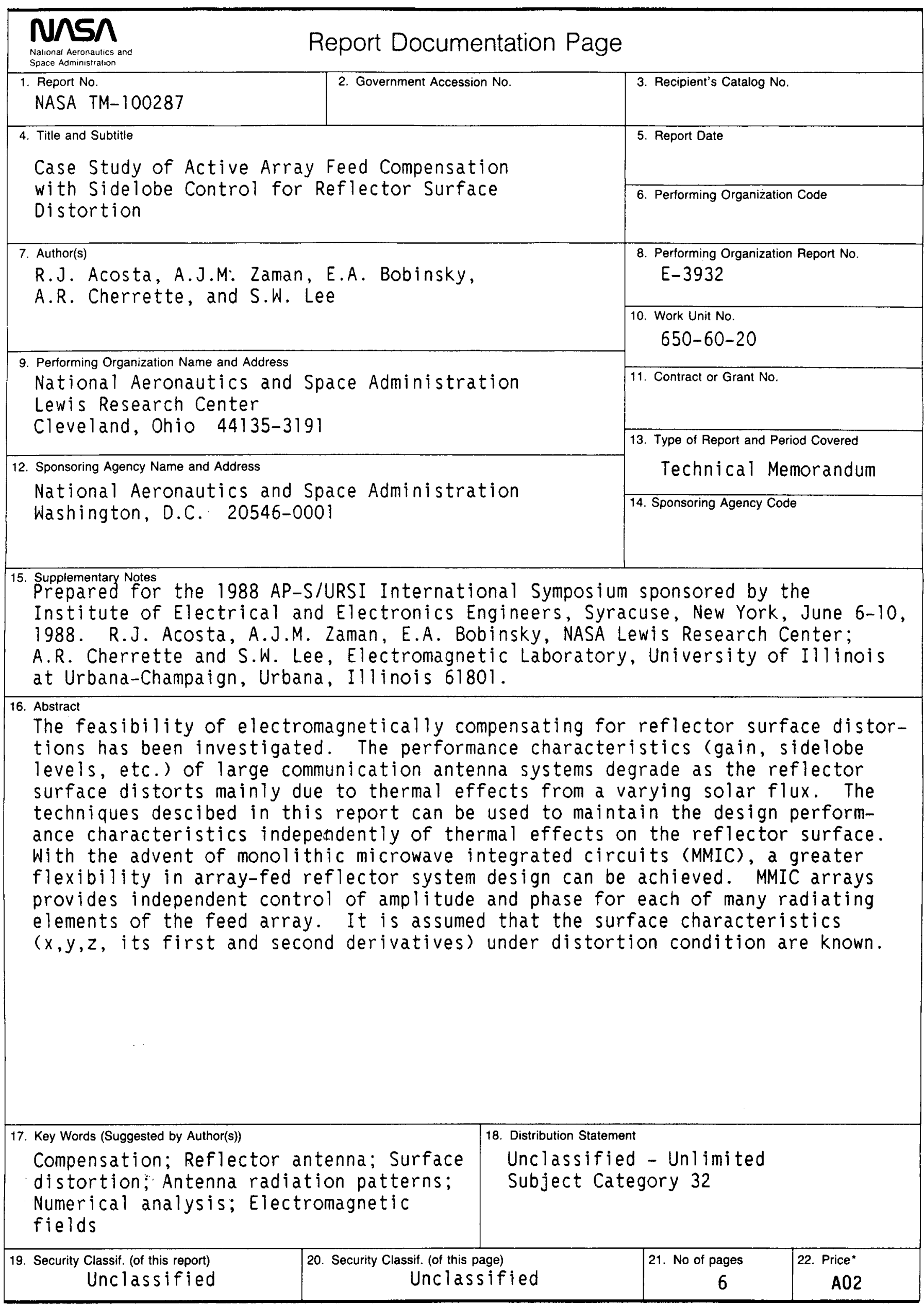

